OPEN ACCESS

Edited by:

Antonio Pisani,

University of Rome Tor Vergata,

Reviewed by:

Benito De Celis Alonso,

BUAP, Mexico

Graziella Madeo,

University of Rome Tor Vergata,

Italy

*Correspondence:

Fernando Cendes

fcendes@gmail.com

Specialty section:

This article was submitted to

Movement Disorders,

a section of the journal

Frontiers in Neurology

Received: 11 October 2016

Accepted: 21 December 2016

Published: 13 January 2017

Citation:

Guimarães RP, Arci Santos MC, Dagher A, Campos LS, Azevedo P, Piovesana LG, De Campos BM, Larcher K, Zeighami Y, Scarparo Amato-Filho AC, Cendes F and D'Abreu ACF (2017) Pattern of Reduced Functional Connectivity and Structural Abnormalities in Parkinson's Disease: An Exploratory Study. Front. Neurol. 7:243.

doi: 10.3389/fneur.2016.00243

\section{Pattern of Reduced Functional Connectivity and Structural Abnormalities in Parkinson's Disease: An Exploratory Study}

\author{
Rachel Paes Guimarães 1,2,3, Maria Cristina Arci Santos², Alain Dagher ${ }^{3}$, \\ Lidiane Soares Campos', Paula Azevedo', Luiza Gonzaga Piovesana', \\ Brunno Machado De Campos², Kevin Larcher ${ }^{3}$, Yashar Zeighami3, \\ Augusto C. Scarparo Amato-Filho ${ }^{4}$, Fernando Cendes ${ }^{1,2 *}$ and \\ Anelyssa Cysne Frota D'Abreu ${ }^{1,2}$
}

\begin{abstract}
'Department of Neurology, University of Campinas, Campinas, Brazil, ${ }^{2}$ Laboratory of Neuroimaging, University of Campinas, Campinas, Brazil, ${ }^{3}$ Montreal Neurological Institute, Brain Imaging Center, McGill University, Montreal, QC, Canada, ${ }^{4}$ Department of Radiology, University of Campinas, Campinas, Brazil
\end{abstract}

Background: MRI brain changes in Parkinson's disease (PD) are controversial.

Objectives: We aimed to describe structural and functional changes in PD.

Methods: Sixty-six patients with PD (57.94 \pm 10.25 years) diagnosed according to the UK Brain Bank criteria were included. We performed a whole brain analysis using voxel-based morphometry (VBM-SPM 8 software), cortical thickness (CT) using CIVET, and resting-state fMRI using the Neuroimaging Analysis Kit software to compare patients and controls. For VBM and CT we classified subjects into three groups according to disease severity: mild PD [Hoehn and Yahr scale (HY) 1-1.5], moderate PD (HY 2-2.5), and severe PD (HY 3-5).

Results: We observed gray matter atrophy in the insula and inferior frontal gyrus in the moderate PD and in the insula, frontal gyrus, putamen, cingulated, and paracingulate gyri in the severe groups. In the CT analysis, in mild PD, cortical thinning was restricted to the superior temporal gyrus, gyrus rectus, and olfactory cortex; in the moderate group, the postcentral gyrus, supplementary motor area, and inferior frontal gyrus were also affected; in the severe PD, areas such as the precentral and postentral gyrus, temporal pole, fusiform, and occipital gyrus had reduced cortical thinning. We observed altered connectivity at the default mode, visual, sensorimotor, and cerebellar networks.

Conclusion: Subjects with mild symptoms already have cortical involvement; however, further cerebral involvement seems to follow Braak's proposed mechanism. Similar regions are affected both structurally and functionally. We believe the combination of different MRI techniques may be useful in evaluating progressive brain involvement and they may eventually be used as surrogate markers of disease progression.

Keywords: Parkinson's disease, neuroimaging, cortical thickness, functional MRI, voxel-based morphometry 


\section{HIGHLIGHTS}

- Subjects with mild symptoms already have cortical involvement; however, further cerebral involvement seems to follow Braak's proposed mechanism.

- Similar brain regions are affected both structurally and functionally, such as temporal, frontal, and occipital gyri; however, it is still unclear if functional alterations are consequence or direct cause of structural ones or if they occur simultaneously.

\section{INTRODUCTION}

Parkinson's disease (PD) is a progressive neurodegenerative disorder (1). Considering the variability of symptoms and the still unknown etiology, MRI-based studies are important to better understand disease physiopathology.

Voxel-based morphometry (VBM) (2) assesses gray matter (GM) density or volume, voxel by voxel. A meta-analysis revealed GM atrophy in the left inferior frontal gyrus, left superior temporal gyrus and left insula in patients with idiopathic PD (3). Only two studies evaluated disease progression as measured by the Hoehn and Yahr scale (HY), and their outcomes differed. One revealed minimal statistically significant GM reduction (4), while the other described atrophy in olfactory-related regions (5).

Cortical thickness (CT) analysis is needed to assess cortical surface properties (6) for it allows subvoxel precision, as thickness values are assigned to individual vertices (7). CT is useful to investigate subtle cortical changes in the brain (8). Previous studies using CT in PD demonstrated widespread alterations (9-11), nonetheless a clear pattern was not established.

Resting-state functional MRI (rs-fMRI) evaluates regional and neuronal circuitry at rest (12), as it measures functional connectivity (FC) in spatially distinctive regions (13). rs-fMRI studies in PD found altered connectivity in the cerebellum, primary motor cortex, supplementary motor area (SMA), dorsolateral and prefrontal cortex, and putamen (4).

Wu et al., using FC global measure through graphical analysis, found increased coupling in the cortex and cerebellum and primary motor cortex, and decreased FC in SMA, dorsolateral and prefrontal cortex, and putamen. It is noteworthy that these results are consistent with the altered pattern of metabolic brain activity found in PD using positron emission tomography (14). Decreased FC in a circuit connecting posterior putamen with the inferior parietal cortex as well as an increased FC between subthalamic nuclei and cortical motor areas were also described, indicating abnormalities in sensorimotor integration and suggesting that some symptoms, such as tremor, may be related to an abnormal coupling of these areas $(15,16)$.

Alterations in CT have also been demonstrated in PD patients in multiple brain regions, such as occipital, temporal, and frontal cortices $(10,17)$. Pereira et al. demonstrated a widespread cortical thinning in lateral occipital, parietal and temporal, frontal and premotor regions.

Cortical thickness measure is a valuable tool for it allows subvoxel precision because thickness values are assigned to individual vertices instead of voxels $(7,9)$.
Despite the progress obtained in the last 30 years trying to comprehend PD pathophysiology, several questions remain.

In general, neuroimaging studies in $\mathrm{PD}$ compare patients with or without certain disorders, such as dementia and depression, but do not consider associated clinical markers like motor symptoms severity, other non-motor symptoms, or the presence of motor complications $(10,18)$.

Furthermore, despite the large number of validated scales for clinical evaluation in PD patients, there are few studies correlating them with MRI findings.

One of the greatest challenges nowadays in $\mathrm{PD}$ is the absence of biomarkers for disease diagnosis in premotor stages and differential diagnosis with other neurodegenerative causes of parkinsonism. Thus, our purpose was to systematically evaluate patients with PD from clinical and neuroimaging data using rs-fMRI and CT analyzes. However, our focus was to generate hypotheses based in this preliminary study, and a longitudinal study is already under way in order to confirm the findings in this paper.

\section{MATERIALS AND METHODS}

Cross-sectional study was conducted at the Neuroimaging Laboratory-Unicamp University Hospital and McConnel Brain Imaging Center-Montreal Neurological Institute (MNI) (McGill University). The State University of Campinas Ethics Committee approved the study, and all individuals signed an informed written consent prior to any research related procedure. All images were acquired at UNICAMP University Hospital and MRI analysis was performed at MNI.

\section{Subjects}

Sixty-six patients $(57.94 \pm 10.25$ years $)$ (43 men) older than 30 years with PD, diagnosed according to the UK Parkinson's Disease Society Brain Bank criteria (19) were consecutively recruited from the Movement Disorders Outpatient Clinic of the University Hospital (UNICAMP), Brazil, and underwent MRI (Table 1). In 46, we performed the whole clinical protocol, including the clinical scales. An experienced neurologist specialized in Movement Disorders assessed all patients.

Evaluation consisted of a standardized questionnaire regarding sex, age, age at disease onset, family history, professional history, environmental exposure to risk factors, and medication used, as well as the application of the Unified Parkinson Disease Rating Scale (UPDRS), HY, Scales for Outcomes in Parkinson's Disease-Cognition (SCOPA-COG), Schwab and England Activities of Daily Living, and Non-Motor Symptom Assessment Scale (NMSS) (Table 1). All patients were on medication on clinical assessment and the MRI acquisition.

We also included 40 healthy controls (HC), with no history of neurological disorders, no family history of $\mathrm{PD}$, and a normal neurological examination (mean age $57.60 \pm 10.77$ ).

For VBM and CT, we classified subjects into three groups according to disease severity: mild PD (HY 1-1.5), moderate PD (HY 2-2.5), and severe PD (HY 3-5) (Table 1).

We performed VBM analysis in 66 patients and $40 \mathrm{HC}$. Eight patients were excluded from the CT analysis due to excessive 
TABLE 1 | Demographic data from all Parkinson's disease (PD) patients, mild PD, moderate PD, and severe PD.

\begin{tabular}{|c|c|c|c|c|c|c|c|c|}
\hline \multirow[b]{2}{*}{ Clinical data } & \multicolumn{2}{|c|}{ All PD } & \multicolumn{2}{|c|}{ Mild PD } & \multicolumn{2}{|c|}{ Moderate PD } & \multicolumn{2}{|c|}{ Severe PD } \\
\hline & Mean & SD & Mean & SD & Mean & SD & Mean & SD \\
\hline Age & 59.33 & 9.8 & 59.31 & 9.60 & 58.5 & 10.69 & 62.07 & 8.4 \\
\hline Education & 6.9 & 4.7 & 8.05 & 4.30 & 7.05 & 5.01 & 4.84 & 3.3 \\
\hline Unified Parkinson's Disease Rating Scale (UPDRS) & 35.74 & 18.47 & 20.94 & 8.86 & 34 & 13.38 & 63.21 & 17.15 \\
\hline 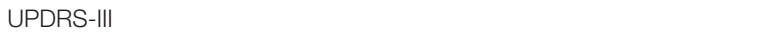 & 16.93 & 8.23 & 10.88 & 78 & 16.2 & 5.95 & 28.35 & 8.88 \\
\hline Hoehn and Yahr scale & 2.8 & 1.26 & 1.25 & 0.25 & 2.57 & 0.3 & 4.42 & 0.51 \\
\hline Scales for Outcomes in Parkinson's Disease - Cognition (SCOPA-COG) & 19.24 & 6.8 & 21.66 & 3.77 & 19.69 & 7.18 & 13.28 & 7.15 \\
\hline SCOPA-CP & 3.6 & 7.8 & 1.55 & 1.72 & 2.77 & 2.37 & 10.07 & 18.63 \\
\hline NMSS & 69.57 & 48.19 & 42.72 & 34.97 & 71.61 & 46.95 & 96.53 & 52.43 \\
\hline SCHWAB (\%) & 73 & 22 & 88 & 8 & 76 & 15 & 34 & 22 \\
\hline Time of disease & 7 & 6.43 & 2.5 & 4.08 & 7 & 6.62 & 12.14 & 5.31 \\
\hline Medication & 5.4 & 5.7 & 2.5 & 1.9 & 3 & 2.6 & 9.5 & 6.8 \\
\hline
\end{tabular}

head movement or the presence of artifacts. The fMRI acquisition protocol was included after the initial recruitment, so only 48 patients and $33 \mathrm{HC}$ had available images.

\section{MRI Acquisition}

Patients and HC underwent the same MRI acquisition protocol on a 3-T Philips Achieva; MRI scanner at UNICAMP:

(1) Echo-planar imaging sequences of $6 \mathrm{~min}$ with isotropic voxel of $3 \mathrm{~mm} \times 3 \mathrm{~mm} \times 3 \mathrm{~mm}$, no gap, 39 slices, FOV $=240 \mathrm{~mm} \times 240 \mathrm{~mm}, \mathrm{TE}=30 \mathrm{~ms}, \mathrm{TR}=2000 \mathrm{~ms}$, flip angle $=90^{\circ}$, and 180 volumes (dynamics), in a 6-min scan;

(2) Volumetric (3D) T1 WI, acquired in the sagittal plane with isotropic voxel with $1 \mathrm{~mm}^{3}$, no gap, flip angle $=35^{\circ}$, $\mathrm{TR}=7.1 \mathrm{~ms}, \mathrm{TE}=3.2 \mathrm{~ms}, \mathrm{FOV}=240 \mathrm{~mm} \times 240 \mathrm{~mm}$, in a 6-min scan.

\section{VBM Analysis}

We used the VBM8 toolbox of the statistical parametric mapping (SPM8) ${ }^{1}$ and the Diffeomorphic Anatomical Registration Exponentiated Lie Algebra (Dartel) software on Matlab R2012b platform to process and analyze the images. VBM allows a voxelwise comparison of local GM differences between two groups. VBM procedure involves the segmentation of the original structural MRI images in native space in GM, white matter (WM), and cerebrospinal fluid (CSF) tissues, followed by GM and WM images normalization to templates in stereotactic space to acquire optimized normalization parameters, which are applied to the raw images. GM images were smoothed using an 8 - $\mathrm{mm}$ full width at half maximum (FWHM) isotropic Gaussian kernel. Finally, we employed a general linear model (GLM), using age and sex as covariates. We obtained the results showing regions of GM concentration with significant differences between the groups (20). Correction for multiple comparisons used the Random Fields Theory (RFT). In each group analysis, a homogeneity test using covariance led to the exclusion of: five patients (PD versus $\mathrm{HC}$ ); one patient and one $\mathrm{HC}$ (mild PD versus $\mathrm{HC}$ ); three patients

${ }^{1}$ http://www.fil.ion.ucl.ac.uk/spm. and one $\mathrm{HC}$ (moderate PD versus $\mathrm{HC}$ ); and five patients and one $\mathrm{HC}$ (severe $\mathrm{PD}$ versus $\mathrm{HC}$ ). The results were displayed using SPM8 and xjView (Human Neuroimaging Lab, Baylor College of Medicine, Houston, TX, USA). We assessed group comparisons by SPM using the family wise error (FWE) at a threshold of $p<0.05$, covariates were sex and age, with an extent threshold of $K=20$ voxels and clusters $>30$ voxels. Brain areas were localized according to the automated anatomical labeling available on The Online Brain Atlas Reconciliation Tool (21).

\section{CT Analysis}

Excessive head movement or the presence of artifacts led to the exclusion of subjects. We used the fully automated pipeline CIVET (version 1.1.10; MNI at McGill University, Montreal, QC, Canada) to estimate CT. Since the fMRI acquisition protocol was also included after the initial recruitment, 48 patients and $33 \mathrm{HC}$ had available images.

In summary, it consists of: correction for magnetic inhomogeneity, skull stripping, linear, and non-linear registration of the native image to the symmetric ICBM 152 template; tissue classification into GM, WM, and CSF, corrected for the partial volume effect; GM and WM surfaces are extracted using the Laplacian map; thickness is calculated at each vertex using the $t$ - link metric method. CT is estimated as the distance, in millimeters, between WM and GM surfaces at each vertex. More details on this technique can be found on the CIVET website. ${ }^{2}$ For this analysis, we regressed out age. We also correlated scales scores with CT from all PD patients.

After that, we stratified patients into mild $(n=16)$, moderate $(n=21)$, and severe PD $(n=11)$ and compared them to HC. Statistical analysis was performed in Matlab (R2008b, The Mathworks, Natick, MA, USA), using the SurfStat toolbox. ${ }^{3}$ We performed a GLM to describe CT as a combination of demographic variables such as age, group (PD or HC), and sex. We used $p<0.05$ (RTF). 


\section{rs-fMRI Analysis}

The fMRI data were preprocessed using the Neuroimaging Analysis Kit (NIAK) release 0.7 (22). ${ }^{4}$ The first three volumes of each run were omitted to allow the magnetization to reach equilibrium. Each fMRI dataset was corrected for inter-slice differences in acquisition time and the parameters of a rigid-body motion were estimated for each time frame. Rigid-body motion was then estimated between runs. The median volume of the first run for each subject was co-registered with a T1 individual scan (23), which was itself non- linearly transformed to MNI space, using the latest version of the ICBM152 template (24). The transformations fMRI-to-T1 and T1-to-stereotaxic were concatenated, to resample the functional volumes into MNI space at a 3-mm isotropic resolution. The "scrubbing" method of Power et al. (25), was used to remove volumes with excessive motion. The fMRI volumes were spatially smoothed with a 6-mm isotropic Gaussian blurring kernel. A more detailed description of the pipeline can be found at: https://github. com/ulrikls/niak. We performed a Bootstrap analysis of stable clusters (BASC). It consists in studying the stability of networks detected with iterative operations of clustering. The resulting stability matrix is used in a clustering procedure, which derives so-called stable clusters. BASC is first applied on individual fMRI time series, and applying a Circular Block Bootstrap to fMRI time series derives replications of clustering. A stability matrix is derived for each subject. The average individual stability matrix is then computed and a hierarchical clustering is applied on it. This allows defining group-level clusters that maximize the average probability of being clustered at the individual level. By bootstrapping the subject stability matrices, the group-level stability matrix is derived. A hierarchical clustering is finally applied on the group stability matrix to define stable group clusters. For each brain region, the average stability of that region with every other region in the cluster of the seed can be derived.

We excluded patients with excessive head movement, since it could result in spurious observations; however, little head movement was accepted (frame displacement lower than 0.2).

Statistical analysis was performed in Matlab (R2008b, The Mathworks, Natick, MA, USA). We performed a GLM to describe connectivity as a combination of variables such as age, group (PD or HC), sex, with head motion as a covariate. We selected a $p<0.05$.

\section{Statistical Analysis}

For statistical analysis, we used STATA 13.1 version. We compared baseline demographic characteristic using a one-way Analysis of Variance, without covariates, followed by a post hoc Sidak test. For categorical variables, we performed a chi-square test. For correlation analyses, we perform a GLM, including the scales scores as independent variables and sex and age as covariates. Level of significance was established at $p<0.05$.

${ }^{4}$ https://github.com/ulrikls/niak.

\section{RESULTS}

\section{Voxel-Based Morphometry}

Mild PD $(n=14)$ versus HC: there was no GM reduction in any region (FWE $p<0.05$ ) (Figure 1; Table 2). Moderate PD $(n=31)$ versus HC: VBM detected GM loss in the left insula and left inferior frontal gyrus, opercular part (FWE $p<0.05)$. Severe PD $(n=16)$ versus HC: we observed GM atrophy in the left insula, left inferior frontal gyrus (opercular part), left putamen, left and right medial superior frontal gyrus, left and right anterior cingulate and paracingulate gyri, and right median cingulated, and paracingulate gyri (FWE $p<0.05)$.

\section{Cortical Thickness}

Mild PD $(n=16)$ versus HC: CT analysis revealed cortical thinning in the left superior temporal gyrus, left gyrus rectus, and left olfactory cortex (RFT $p<0.05)$. Moderate PD $(n=21)$ versus $\mathrm{HC}$ : the areas with cortical thinning were the right postcentral gyrus, right SMA, and right inferior frontal gyrus (triangular and opercular parts) (RFT $p<0.05$ ) (Figure 1; Table 2). Severe PD $(n=11)$ versus HC: CT analysis revealed cortical thinning in the left inferior frontal gyrus, left precentral and postcentral gyrus, left SMA, left inferior frontal gyrus (triangular part), left gyrus rectus, right temporal pole, right fusiform gyrus, right middle temporal gyrus, and right occipital gyrus (RFT $p<0.05$ ). We found a negative correlation between UPDRS and CT in superior temporal gyrus, pre and post central gyrus, superior and inferior frontal gyrus, SMA, occipital gyrus, and gyrus rectus. The same negative correlation was found regarding UPDRS-III in superior and medial temporal gyrus, lingual gyrus, parahipocampal gyrus, SMA, and medial temporal gyrus $(p<0.05)$. SCOPA and NMSS scores did not correlate with CT values.

\section{Resting-State Functional MRI}

The final sample was too small to further subdivide, so we performed a whole brain analysis, without an a priori assumption (Figure 2; Table 3). Our main objective was to demonstrate by cluster analysis that the resting-state abnormalities occur in the whole brain in PD patients. Based on data provided by the BASC pipeline, we selected the scale with 70 clusters as the most stable one and examined the networks found at this scale (22). We found reduced connectivity within visual, sensorimotor, DMN, and cerebellum networks.

\section{DISCUSSION}

We evaluated PD patients using VBM, CT, and rs-fMRI analysis without an a priori assumption. GM volume and CT are the two most widely used measures for detecting GM morphometric changes, but they measure different aspects of the brain, hence the importance of performing both methods (8). We observed a certain concurrence between VBM and CT findings within each stage.

One region with decreased $\mathrm{CT}$ in mild $\mathrm{PD}$ was the left olfactory cortex, which receives sensory information from the 


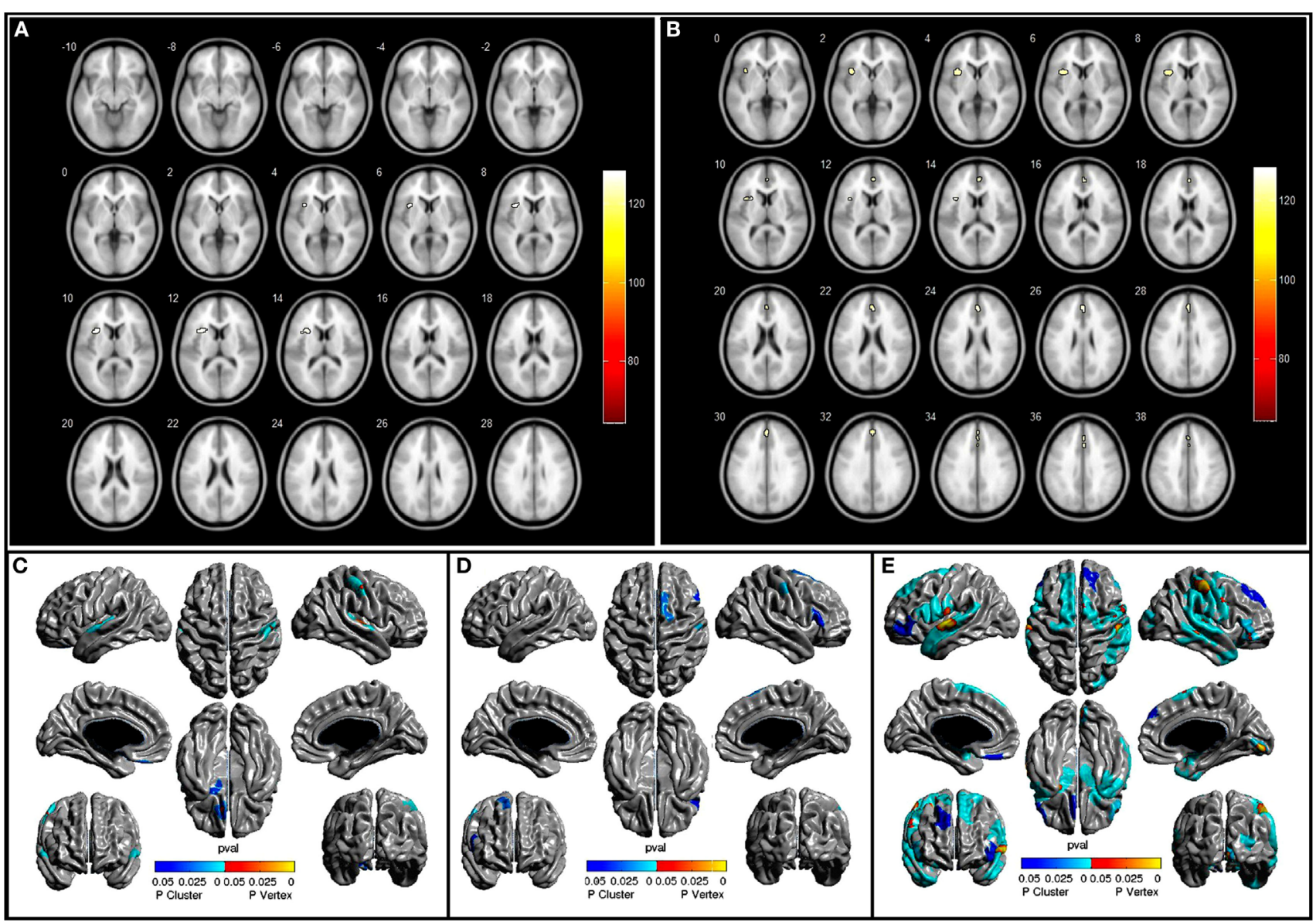

FIGURE 1 | (A,B) show areas with atrophy in moderate PD and severe PD patients when compared to healthy controls (HC), $p<0.05$ FEW, (C-E) show area with cortical thinking in mild PD, moderate PD, and severe PD, respectively, when compared to HC $p<0.5$, Random Fields Theory.

olfactory bulb (26), which is in keeping with the olfactory loss as one of the early non-motor manifestations of PD as well as with previous findings of Lewy pathology in anterior olfactory nucleus and olfactory bulb (27). We did not find the same alteration using VBM; however, CT analyses are more sensitive than VBM for identifying regional cortical thinning associated with PD because VBM merges information about morphology, size, and position and the final measures average information about thickness and cortical folding (2), possibly resulting in less sensitivity.

Olfactory loss is an important non-motor manifestation in PD, and it seems to be present years before diagnosis (28). We could not find an association between NMSS scores and CT; however, we used the scale total score, and possibly, an assessment of each scale domain is more appropriate for $\mathrm{PD}$, especially in longitudinal analysis. Previous studies demonstrated a correlation between amygdala and hippocampus volume with depression and anxiety in PD patients (29); however, these symptoms are non-specific and can be present in normal aging (30).

The widely known and accepted Braak's stage postulates that the Lewy body (LB) deposition in PD progresses in an ascending fashion (31). In stages 1 and 2 , it is essentially restricted to the medulla oblongata. In stages 3 and 4 , the involvement is mostly observed in the brain stem without cortical lesions (stage 3 ) or with initial deposition at the anteromedial temporal mesocortex (stage 4), while in stages 5 and 6, there is inexorable cortical involvement. Recent studies suggest the possibility that $\alpha$-synuclein is a prion-like protein and that PD is a prion-like disease, a theory compatible with Braak's staging system (32). Other pathological studies, however, have clearly challenged the generalizability of this hypothesis, and as far as we know, it has not been demonstrated by in vivo studies (33).

Considering our findings, even patients with mild motor symptoms, already have cortical involvement (possibly stage 4/5), while we did not observe significant atrophy in the brainstem. We believe this is due to a compensatory mechanism of the brain, where brain alterations are already present but there is still no clinical symptoms.

The further progression seems to follow the previously described pattern. It is worth mentioning, nevertheless, that while the Braak's stage takes LB depositions into consideration, imaging analyses are mostly concerned with GM loss. Hence, some of the differences in the results may reflect the variable 
TABLE 2 | Areas with reduced cortical thickness (CT) on the left and GM atrophy on the right of mild Parkinson's disease (PD) versus healthy controls (HC), moderate PD versus HC and severe PD versus HC $(p<0.05)$.

\begin{tabular}{|c|c|c|c|c|c|}
\hline \multicolumn{3}{|c|}{ CT } & \multicolumn{3}{|c|}{ Voxel-based morphometry } \\
\hline Coordinates $(x$ y z) & Areas & $p$ [random fields Theory (RFT)] & Coordinates ( $x$ y $z$ ) & Areas & $p$ (RFT) \\
\hline \multicolumn{6}{|l|}{ Mild PD } \\
\hline$-48-110$ & L superior Temporal Gyrus & $<0.05$ & - & - & - \\
\hline$-931-21$ & Gyrus rectus & $<0.05$ & - & - & - \\
\hline$-219-18$ & L superior frontal gyrus & $<0.05$ & - & - & - \\
\hline$-166-16$ & L olfactory cortex & $<0.05$ & - & - & - \\
\hline $55-1646$ & R post central gyrus & $<0.05$ & - & - & - \\
\hline \multicolumn{6}{|l|}{ Moderate PD } \\
\hline 14169 & Supplementary motor area (SMA) & $<0.05$ & -30149 & $\mathrm{~L}$ insula & $<0.05$ \\
\hline 53259 & Inferior frontal gyrus & $<0.05$ & -30149 & $L$ inferior frontal gyrus & $<0.05$ \\
\hline 16071 & R superior frontal Gyrus & $<0.05$ & & & \\
\hline \multicolumn{6}{|l|}{ Severe PD } \\
\hline-41219 & $L$ inferior frontal gyrus & $<0.05$ & -35143 & $\mathrm{~L}$ insula & $<0.05$ \\
\hline$-64-1217$ & L postcentral gyrus & $<0.05$ & -35143 & $L$ inferior frontal gyrus & $<0.05$ \\
\hline $35-10-38$ & Fusiform gyrus & $<0.05$ & -35143 & L putamen & $<0.05$ \\
\hline$-29-1570$ & $L$ precentral gyrus & $<0.05$ & 04125 & R superior frontal gyrus & $<0.05$ \\
\hline$-9-170$ & LSMA & $<0.05$ & 04125 & Anterior cingulum & $<0.05$ \\
\hline$-14-1267$ & L superior frontal gyrus & $<0.05$ & 03336 & L superior frontal gyrus & $<0.05$ \\
\hline$-7-1171$ & Paracentral lobule & $<0.05$ & - & - & - \\
\hline $514-34$ & R medial temporal gyrus & $<0.05$ & - & - & - \\
\hline $2514-40$ & R superior temporal gyrus & $<0.05$ & - & - & - \\
\hline
\end{tabular}

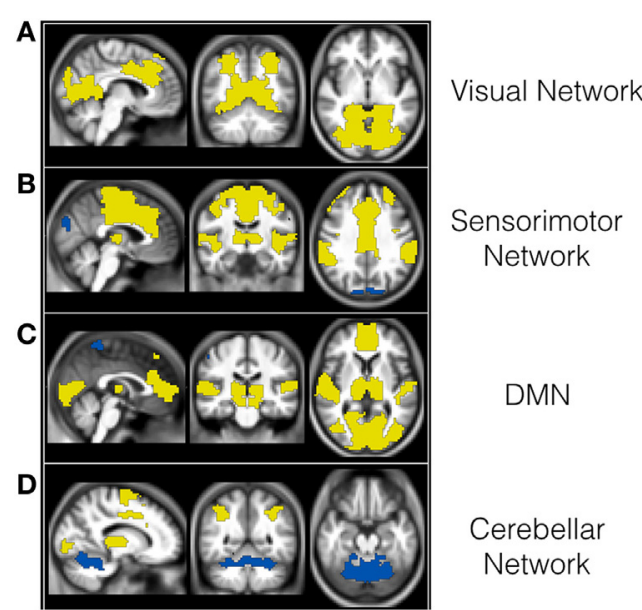

FIGURE 2 | Reduced functional connectivity (FC) within visual (A), sensorimotor (B), DMN (C), and cerebellum networks (D) in PD patients when compared to controls. The areas in yellow have reduced FC with areas in blue in PD patients compared to controls at $p$ level 0.05 .

under study, and its measurement and not the validity of the findings. VBM is also not a particular sensitive tool for posterior fossa analysis, and the presence of LB does not necessarily imply measurable cortical volume change. Moreover, previous studies suggested that synaptic dysfunction can occur without the presence of Lewy bodies per se and that using the presence of LB to measure pathological progression might not be enough to predict the spread of disease (34).

Nevertheless, the importance of these findings is clear. Pathology is far advanced throughout the brain when patients are actually diagnosed with the disease. Even though this has been suggested by the knowledge and identification of non-motor symptoms in the premotor phase (35), it had not been universally demonstrated in idiopathic PD (4). This is a fundamental issue when addressing neuroprotective therapies. Since cortical areas are involved at the onset of motor symptoms, even patients with very early disease may not be the ideal candidates for clinical studies. The process of brain pathology may be so advanced that those therapies may no longer work, or possibly longer periods of observation would be necessary to address neuroprotection. Also, PD has diverse clinical manifestations and possibly different PD subtypes may have different underlying pathological patterns. Future studies may consider studying the posterior fossa in detail in subjects with mild or very early disease, such as the SUIT tool for VBM (36).

Since our study is a cross-sectional analysis of disease stages, we can only infer progression of the cerebral involvement as the clinical manifestations worsens. Previous longitudinal studies using different imaging analysis have suggested that striatal atrophy occurs earlier in the disease process, while cortical GM loss is restricted to later stages, reduced overall gyrification, and bilaterally in the inferior parietal, postcentral, precentral, superior frontal, and supramarginal areas was present in patients with disease for less than 1 year, and the rate loss was accelerated as disease progressed (37). Although our subjects presented some homogeneity in their mean clinical scale scores per group (with lower scores in the mild group, intermediate in the moderate group, and higher scores in the severe group in the motor and non-motor scales), we classified them solely based on their HY score. The HY is a well-accepted scale, as it correlates with imaging studies of dopamine loss and with quality of life, motor impairment, and disability scales (38). However, it does not offer 
TABLE 3 | Areas with reduced functional connectivity in Parkinson's disease patients when compared to healthy controls within clusters ( $p<0.05)$.

\begin{tabular}{|c|c|c|c|c|}
\hline Cluster & Coordinate $(x y z)$ & Networks & Areas with altered connectivity & $p$-Value \\
\hline 16 & $-658-2$ & Visual & $\begin{array}{l}\text { Parhippocampal gyrus } \\
\text { Lingual gyrus } \\
\text { Occipital pole } \\
\text { Superior parietal lobule } \\
\text { Precentral gyrus } \\
\text { Supramarginal gyrus }\end{array}$ & $\begin{array}{l}<0.05 \\
<0.05 \\
<0.05 \\
<0.05 \\
<0.05 \\
<0.05\end{array}$ \\
\hline 38 & -31034 & Sensorimotor & $\begin{array}{l}\text { Thalamus } \\
\text { Insula } \\
\text { Superior temporal gyrus } \\
\text { Precentral gyrus } \\
\text { Inferior frontal gyrus } \\
\text { Middle frontal gyrus } \\
\text { Parietal lobule } \\
\text { Supramarginal gyrus } \\
\text { Cingulate Gyrus }\end{array}$ & $\begin{array}{l}<0.05 \\
<0.05 \\
<0.05 \\
<0.05 \\
<0.05 \\
<0.05 \\
<0.05 \\
<0.05 \\
<0.05\end{array}$ \\
\hline 66 & $0-224$ & $\mathrm{DMN}$ & $\begin{array}{l}\text { Thalamus } \\
\text { Occipital lobe } \\
\text { Lingual gyrus } \\
\text { Anterior cingulate } \\
\text { Cingulate gyrus } \\
\text { Superior frontal gyrus } \\
\text { Superior temporal gyrus }\end{array}$ & $\begin{array}{l}<0.05 \\
<0.05 \\
<0.05 \\
<0.05 \\
<0.05 \\
<0.05 \\
<0.05\end{array}$ \\
\hline 69 & $0-67-14$ & Cerebellar & $\begin{array}{l}\text { Lingual gyrus } \\
\text { Thalamus } \\
\text { Cingulate Gyrus } \\
\text { Precentral gyrus } \\
\text { Frontal gyrus } \\
\text { Angular gyrus } \\
\text { Frontal pole } \\
\text { Occipital cortex } \\
\text { Brainstem }\end{array}$ & $\begin{array}{l}<0.05 \\
<0.05 \\
<0.05 \\
<0.05 \\
<0.05 \\
<0.05 \\
<0.05 \\
<0.05 \\
<0.05\end{array}$ \\
\hline
\end{tabular}

any direct information regarding non-motor issues. It should be also noted that the age in the groups were inadvertently similar. We did not pair the three samples based on age. So, more severe subjects had longer disease duration and an earlier onset. Patients with earlier onset usually have a less aggressive disease, compared to those with a later onset (39). It has been previously described that brain alteration in patients with earlier onset and long disease duration fit the Braak's model, while in patients with later onset and short disease duration it did not occur (40). Thus, we may have underestimated the degree of atrophy in the severe group and a longitudinal approach, taking age of onset and possibly clinical subtypes is the next step in this exploratory study.

Functional connectivity was decreased within visual, sensorimotor, DMN, and cerebellar networks. Although there is overwhelming evidence that several networks are altered in PD (41-43), the results from previous studies are controversial. The methods used are as diverse as the results. They differ in subject samples, type of MRI analysis, and the ROI chosen, making them difficult to compare. Most studies with fMRI use the independent component analysis (ICA) technique, which is based on the theory that the fMRI signal of each voxel represents a linear mixture of signals. These are separated by statistical analysis of independent signals, and finally, the brain regions with the same independent signals are grouped together as separate components (44). ICA, however, finds functional networks in data randomly generated, since the algorithm used is an optimization procedure, and values can vary due to the chosen threshold and variability among patients (45). Conversely, the BASC method provides a fully automated alternative that applies to an arbitrarily large number of networks (22). BASC renders more robust results since it uses the growth region to find networks and observes the replicability of these through the clusters stability analysis.

Unfortunately, our sample was relatively small and heterogeneous, and we were unable to classify the subjects into the same groups in the rs-fMRI analysis. We can only conclude from our analysis that there is widespread decreased FC in PD, and this correlates with the regions in which we observed atrophy and decreased CT in our structural analysis. Different patterns of reduced connectivity may explain differences in clinical presentation within patients with PD, such as dyskinesias (46) and behavioral differences (47). In the future, we may be able to study the pattern of FC in the individual patient, and from the configuration observed, there is a chance we may be able to predict how the disease will progress, or select individualized targets for neurosurgical procedures.

\section{CONCLUSION}

In summary, we used validated, complementary, and welldocumented techniques to assess brain alterations in PD. We 
confirmed that subjects with mild symptoms already have cortical involvement and similar regions are affected both structurally and functionally. Nevertheless, future longitudinal studies are needed to further comprehend all the mechanisms contributing to PD and its clinical subtypes.

\section{ETHICS STATEMENT}

This study was carried out in accordance with the recommendations of Campinas State University ethics committee with written informed consent from all subjects. All subjects gave written informed consent in accordance with the Declaration of Helsinki. The protocol was approved by the Campinas State University ethics committee.

\section{AUTHOR CONTRIBUTIONS}

Research project conception: RG, MS, AD, ACD, and FC. Research project organization: RG, MS, ACD, and FC. Research project execution: RG, MS, LC, LP, PA, AA-F, KL, and YZ. Statistical analysis design: $\mathrm{RG}, \mathrm{MS}, \mathrm{AD}, \mathrm{BC}, \mathrm{KL}$, and $\mathrm{YZ}$. Statistical analysis

\section{REFERENCES}

1. Duncan GW, Khoo TK, Yarnall AJ, O’Brien JT, Coleman SY, Brooks DJ, et al. Health-related quality of life in early Parkinson's disease: the impact of nonmotor symptoms. Mov Disord (2014) 29(2):195-202. doi:10.1002/ mds.25664

2. Ashburner J, Friston KJ. Voxel-based morphometry - the methods. Neuroimage (2000) 11:805-21.

3. Pan PL, Song W, Shang HF. Voxel-wise meta-analysis of gray matter abnormalities in idiopathic Parkinson's disease. Eur J Neurol (2012) 19(2):199-206. doi:10.1111/j.1468-1331.2011.03474.x

4. Agosta F, Canu E, Stojkovic T, Pievani M, Tomic A, Sarro L, et al. The topography of brain damage at different stages of Parkinson's disease. Hum Brain Mapp (2013) 34(11):2798-807. doi:10.1002/hbm.22101

5. Wattendorf E, Welge-Luessen A, Fiedler K, Bilecen D, Wolfensberger M, Fuhr P, et al. Olfactory impairment predicts brain atrophy in Parkinson's disease. J Neurosci (2009) 29(49):15410-3. doi:10.1523/JNEUROSCI.190909.2009

6. Foster NE, Doyle-Thomas KA, Tryfon A, Ouimet T, Anagnostou E, Evans AC, et al. Structural gray matter differences during childhood development in autism spectrum disorder: a multimetric approach. Pediatr Neurol (2015) 53(4):350-9. doi:10.1016/j.pediatrneurol.2015.06.013

7. Fischl B, Dale AM. Measuring the thickness of the human cerebral cortex from magnetic resonance images. Proc Natl Acad Sci U S A (2000) 97(20):11050-5. doi:10.1073/pnas.200033797

8. Kong L, Herold CJ, Zollner F, Salat DH, Lasser MM, Schmid LA, et al. Comparison of grey matter volume and thickness for analysing cortical changes in chronic schizophrenia: a matter of surface area, grey/white matter intensity contrast, and curvature. Psychiat Res (2015) 231(2):176-83. doi:10.1016/j.pscychresns.2014.12.004

9. Pereira JB, Ibarretxe-Bilbao N, Marti MJ, Compta Y, Junque C, Bargallo N, et al. Assessment of cortical degeneration in patients with Parkinson's disease by voxel-based morphometry, cortical folding, and cortical thickness. Hum Brain Mapp (2012) 33(11):2521-34. doi:10.1002/hbm.21378

10. Hanganu A, Bedetti C, Jubault T, Gagnon JF, Mejia-Constain B, Degroot C, et al. Mild cognitive impairment in patients with Parkinson's disease is associated with increased cortical degeneration. Mov Disord (2013) 28(10):1360-9. doi:10.1002/mds.25541

11. Baggio HC, Segura B, Garrido-Millan JL, Marti MJ, Compta Y, Valldeoriola F, et al. Resting-state frontostriatal functional connectivity in Parkinson's execution: RG, MS, BC, and KL. Statistical analysis review and critique: $\mathrm{AD}, \mathrm{ACD}$, and $\mathrm{FC}$. Manuscript preparation and writing of the first draft: RG and MS, review and critique: AD, ACD, FC, and AA-F.

\section{ACKNOWLEDGMENTS}

The authors would like to thank Dr. Friedman for kindly reviewing this manuscript and his valuable suggestions.

\section{FUNDING}

This work was supported by FAPESP (São Paulo Research Foundation), grant numbers: 2012/05286-7, 2011/19958-4 and 2013/03358-3 and by CNPq, grant number 74873/2010-2. RG: Research grant from FAPESP. MS: Research grant from FAPESP. LC: Educational grant from Ipsen. PA: Educational grant from Ipsen. LP: Educational grant from Ipsen. BC: none. KL: none. YZ: none. AA-F: none. AD: none. FC: Supported by grants from FAPESP and CNPq, BRAZIL. ACD: Research grant from FAPESP and $\mathrm{CNPq}$, Brazil. disease-related apathy. Mov Disord (2015) 30(5):671-9. doi:10.1002/ mds. 26137

12. Scholvinck ML, Maier A, Ye FQ, Duyn JH, Leopold DA. Neural basis of global resting-state fMRI activity. Proc Natl Acad Sci U S A (2010) 107(22):10238-43. doi:10.1073/pnas.0913110107

13. Biswal B, Yetkin FZ, Haughton VM, Hyde JS. Functional connectivity in the motor cortex of resting human brain using echo-planar MRI. Magn Reson Med (1995) 34(4):537-41. doi:10.1002/mrm.1910340409

14. Huang C, Mattis P, Tang C, Perrine K, Carbon M, Eidelberg D. Metabolic brain networks associated with cognitive function in Parkinson's disease. Neuroimage (2007) 34:714-23. doi:10.1016/j.neuroimage.2006.09.003

15. Baudrexel S, Witte T, Seifried C, von Wegner F, Beissner F, Klein JC, et al. Resting state fMRI reveals increased subthalamic nucleus-motor cortex connectivity in Parkinson's disease. Neuroimage (2011) 55(4):1728-38. doi:10.1016/j.neuroimage.2011.01.017

16. Ghasemi M, Mahloojifar A. Disorganization of equilibrium directional interactions in the brain motor network of Parkinson's disease: new insight of resting state analysis using granger causality and graphical approach. J Med Signals Sens (2013) 3(2):69-78.

17. Zhang Y, Zhang J, Xu J, Wu X, Zhang Y, Feng H, et al. Cortical gyrification reductions and subcortical atrophy in Parkinson's disease. Mov Disord (2014) 29(1):122-6. doi:10.1002/mds.25680

18. Olde Dubbelink KT, Schoonheim MM, Deijen JB, Twisk JW, Barkhof F, Berendse HW. Functional connectivity and cognitive decline over 3 years in Parkinson disease. Neurology (2014) 83(22):2046-53. doi:10.1212/ WNL.0000000000001020

19. Daniel SE, Lees AJ. Parkinson's disease society brain bank, London: overview and research. J Neural Transm Suppl (1993) 39:165-72.

20. Matsuda H, Mizumura S, Nemoto K, Yamashita F, Imabayashi E, Sato N, et al. Automatic voxel-based morphometry of structural MRI by SPM8 plus diffeomorphic anatomic registration through exponentiated lie algebra improves the diagnosis of probable Alzheimer Disease. AJNR Am J Neuroradiol (2012) 33(6):1109-14. doi:10.3174/ajnr.A2935

21. Tzourio-Mazoyer N, Landeau B, Papathanassiou D, Crivello F, Etard O, Delcroix N, et al. Automated anatomical labeling of activations in SPM using a macroscopic anatomical parcellation of the MNI MRI single-subject brain. Neuroimage (2002) 15(1):273-89. doi:10.1006/nimg.2001.0978

22. Bellec P, Rosa-Neto P, Lyttelton OC, Benali H, Evans AC. Multi-level bootstrap analysis of stable clusters in resting-state fMRI. Neuroimage (2010) 51:1126-39. doi:10.1016/j.neuroimage.2010.02.082 
23. Collins DL, Neelin P, Peters TM, Evans AC. Automatic 3D intersubject registration of MR volumetric data in standardized Talairach space. J Comput Assist Tomogr (1994) 18(2):192-205. doi:10.1097/00004728-19940300000005

24. Fonov V, Evans AC, Botteron K, Almli CR, McKinstry RC, Collins DL, et al. Unbiased average age-appropriate atlases for pediatric studies. Neuroimage (2011) 54(1):313-27. doi:10.1016/j.neuroimage.2010.07.033

25. Power JD, Barnes KA, Snyder AZ, Schlaggar BL, Petersen SE. Spurious but systematic correlations in functional connectivity MRI networks arise from subject motion. Neuroimage (2012) 59(3):2142-54. doi:10.1016/ j.neuroimage.2011.10.018

26. Mizutani Y, Nakamura T, Okada A, Suzuki J, Watanabe H, Hirayama M, et al. Hyposmia and cardiovascular dysautonomia correlatively appear in early-stage Parkinson's disease. Parkinsonism Relat Disord (2014) 20(5):520-4. doi:10.1016/j.parkreldis.2014.02.010

27. Visanji NP, Brooks PL, Hazrati LN, Lang AE. The prion hypothesis in Parkinson's disease: braak to the future. Acta Neuropathol Commun (2013) 1:2. doi:10.1186/2051-5960-1-2

28. Mehta SH, Adler CH. Advances in biomarker research in Parkinson's disease. Curr Neurol Neurosci Rep (2016) 16(1):7. doi:10.1007/s11910-015-0607-4

29. van Mierlo TJ, Chung C, Foncke EM, Berendse HW, van den Heuvel OA. Depressive symptoms in Parkinson's disease are related to decreased hippocampus and amygdala volume. Mov Disord (2015) 30(2):245-52. doi:10.1002/ mds. 26112

30. Bowman FD, Drake DF, Huddleston DE. Multimodal imaging signatures of Parkinson's disease. Front Neurosci (2016) 10:131. doi:10.3389/ fnins.2016.00131

31. Braak H, Del Tredici K, Rub U, de Vos RA, Jansen Steur EN, Braak E. Staging of brain pathology related to sporadic Parkinson's disease. Neurobiol Aging (2003) 24:197-211. doi:10.1016/S0197-4580(02)00065-9

32. Olanow CW, Brundin P. Parkinson's disease and alpha synuclein: is Parkinson's disease a prion-like disorder? Mov Disord (2013) 28(1):31-40. doi:10.1002/ mds. 25373

33. Klingelhoefer L, Reichmann H. Pathogenesis of Parkinson disease - the gutbrain axis and environmental factors. Nat Rev Neurol (2015) 11(11):625-36. doi:10.1038/nrneurol.2015.197

34. Milber JM, Noorigian JV, Morley JF, Petrovitch H, White L, Ross GW, et al. Lewy pathology is not the first sign of degeneration in vulnerable neurons in Parkinson disease. Neurology (2012) 79(24):2307-14. doi:10.1212/ WNL.0b013e318278fe32

35. Kalia LV, Lang AE. Parkinson's disease. Lancet (2015) 386(9996):896-912. doi:10.1016/S0140-6736(14)61393-3

36. Diedrichsen J. A spatially unbiased atlas template of the human cerebellum. Neuroimage (2006) 33(1):127-38. doi:10.1016/j.neuroimage.2006.05.056

37. Lewis MM, Du G, Lee EY, Nasralah Z, Sterling NW, Zhang L, et al. The pattern of gray matter atrophy in Parkinson's disease differs in cortical and subcortical regions. J Neurol (2016) 263(1):68-75. doi:10.1007/s00415-0157929-7

38. Goetz CG, Poewe W, Rascol O, Sampaio C, Stebbins GT, Counsell C, et al. Movement disorder society task force report on the Hoehn and Yahr staging scale: status and recommendations. Mov Disord (2004) 19(9):1020-8. doi: $10.1002 / \mathrm{mds} .20213$

39. Suchowersky O, Reich S, Perlmutter J, Zesiewicz T, Gronseth G, Weiner WJ, et al. Practice parameter: diagnosis and prognosis of new onset Parkinson disease (an evidence-based review) report of the Quality Standards Subcommittee of the American Academy of Neurology. Neurology (2006) 66(7):968-75. doi:10.1212/01.wnl.0000215437.80053.d0

40. Halliday G, Hely M, Reid W, Morris J. The progression of pathology in longitudinally followed patients with Parkinson's disease. Acta Neuropathol (2008) 115(4):409-15. doi:10.1007/s00401-008-0344-8

41. Gottlich M, Munte TF, Heldmann M, Kasten M, Hagenah J, Kramer UM. Altered resting state brain networks in Parkinson's disease. PLoS One (2013) 8(10):e77336. doi:10.1371/journal.pone.0077336

42. Amboni M, Tessitore A, Esposito F, Santangelo G, Picillo M, Vitale C, et al. Resting-state functional connectivity associated with mild cognitive impairment in Parkinson's disease. J Neurol (2015) 262(2):425-34. doi:10.1007/ s00415-014-7591-5

43. Baggio HC, Segura B, Junque C. Resting-state functional brain networks in Parkinson's disease. CNS Neurosci Ther (2015) 21(10):793-801. doi:10.1111/ cns. 12417

44. Calhoun VD, Liu J, Adali T. A review of group ICA for fMRI data and ICA for joint inference of imaging, genetic, and ERP data. Neuroimage (2009) 45(1):S163-72. doi:10.1016/j.neuroimage.2008.10.057

45. Ylipavalniemi J, Vigario R. Analyzing consistency of independent components: an fMRI illustration. Neuroimage (2008) 39(1):169-80. doi:10.1016/j. neuroimage.2007.08.027

46. Herz DM, Haagensen BN, Nielsen SH, Madsen KH, Lokkegaard A, Siebner HR. Resting-state connectivity predicts levodopa-induced dyskinesias in Parkinson's disease. Mov Disord (2016) 31(4):521-9. doi:10.1002/ mds. 26540

47. Vervoort G, Alaerts K, Bengevoord A, Nackaerts E, Heremans E, Vandenberghe $\mathrm{W}$, et al. Functional connectivity alterations in the motor and fronto-parietal network relate to behavioral heterogeneity in Parkinson's disease. Parkinsonism Relat Disord (2016) 24:48-55. doi:10.1016/j.parkreldis.2016.01.016

Conflict of Interest Statement: The authors declare that the research was conducted in the absence of any commercial or financial relationships that could be construed as a potential conflict of interest.

The reviewer GM and handling Editor declared their shared affiliation, and the handling Editor states that the process nevertheless met the standards of a fair and objective review.

Copyright (C) 2017 Guimarães, Arci Santos, Dagher, Campos, Azevedo, Piovesana, De Campos, Larcher, Zeighami, Scarparo Amato-Filho, Cendes and D'Abreu. This is an open-access article distributed under the terms of the Creative Commons Attribution License (CC BY). The use, distribution or reproduction in other forums is permitted, provided the original author(s) or licensor are credited and that the original publication in this journal is cited, in accordance with accepted academic practice. No use, distribution or reproduction is permitted which does not comply with these terms. 